\title{
Advancing TEM Based Biomedical Nanoparticle Characterization: GMP Compliant TEM Workflow in a BSL2 Environment and Automation Using MiniTEM
}

Mathieu Colomb-Delsuc ${ }^{1}$, Johan Härmark ${ }^{1}$, Birgitta Eriksson ${ }^{2}$, Rickard Nordström ${ }^{1}$, Vanessa Carvalho $^{1}$, Gustaf Kylberg ${ }^{3}$ and Ida-Maria Sintorn ${ }^{3}$

1. Electron Microscopy Services, Vironova AB, Stockholm, Sweden.

2. Complyit AB, Stockholm, Sweden.

3. Research and Development, Vironova AB, Stockholm, Sweden.

* Corresponding author: mathieu.colomb-delsuc@vironova.com

Transmission Electron Microscopy (TEM) is routinely used for the characterization of nano-sized particles in life sciences. Among the variety of specimen analyzed, some involve risks and require strict safety procedures when being handled. Working with infectious and pathogenic agents such as some viruses, vaccines or gene therapy vectors for example, implies to operate in contained facilities with a well-controlled environment.

Some of the requirements for working in Biosafety Level 2 (BSL2) conditions, as described by the European Commission guidelines [1], are quite challenging to fulfill within the standard TEM workflow. Consequently, the daily routines need to be adapted in order to combine the challenges of TEM with safety requirements, such as performing precision work with bulky gowning, preparing the specimen and transferring liquid nitrogen inside a facility with a high air-flow, or decontaminating the equipment used for the specimen preparation with corrosive agents. To answer to the BSL2 demands, established standard protocols need to be implemented and validated. This to ensure that the equipment is not damaged, and the safety of the operator is guaranteed, along with reliability of the results. Moreover, access to this type of facility needs to be controlled and provided only to personnel that has specific and documented training for preparing and imaging TEM specimens in such conditions. The set-up of a BSL3 laboratory, requiring even stricter constraints, has previously been described [2].

Construction and establishment of such a BSL2 TEM facility (Figure 1) including a Good Manufacturing Practice (GMP) compliant TEM workflow, proves to be tremendously relevant, especially for e.g., the vaccine and gene therapy domains. In these fields new requirements from the United States Pharmacopeial Convention (USP) and the U.S. Food and Drug Administration (FDA) place stress on investigating the impact of changes on the product during process and product development. New demands in product characterization involve morphological description of the particles present in the formulations, typically nanoparticles like viruses, virus-like particles and adjuvants in the range of $0.01-0.1 \mu \mathrm{m}$. TEM offers the means to answer these questions in a direct way. In addition, when entering the production phase of such products, TEM provides excellent means for production monitoring and quality control. Therefore, being able to prepare and image specimens while respecting the safety guidelines also for BSL2 specimen is a clear need.

In parallel to this work, we have developed a desktop sized transmission electron microscope, the MiniTEM $^{\mathrm{TM}}$. The system operates at a low voltage $(25 \mathrm{keV})$, and has reduced complexity and maintenance costs. The idea behind MiniTEM was to develop a system that is easy to operate also for users with limited experience in electron microscopy, that is compact, and easy to place and deploy, and 
offers a high degree of automation for imaging and analysis. These features designed for convenient user unbiased analysis, also makes it suitable for BSL2 facilities.

The challenges and hurdles that were overcome to implement a GMP workflow in a BSL2 lab for the analysis of biological specimen with TEM will be presented and discussed, together with a description of MiniTEM ${ }^{\mathrm{TM}}$ system, illustrated with case studies demonstrating its analytical capacities and application domains.

\section{References:}

[1] European law website, https://eur-lex.europa.eu/legal-content/EN/TXT/?uri=CELEX:32000L0054 (accessed February 22, 2019)

[2] MB Sherman et al., Journal of Structural Biology 181 (2013), p. 223.
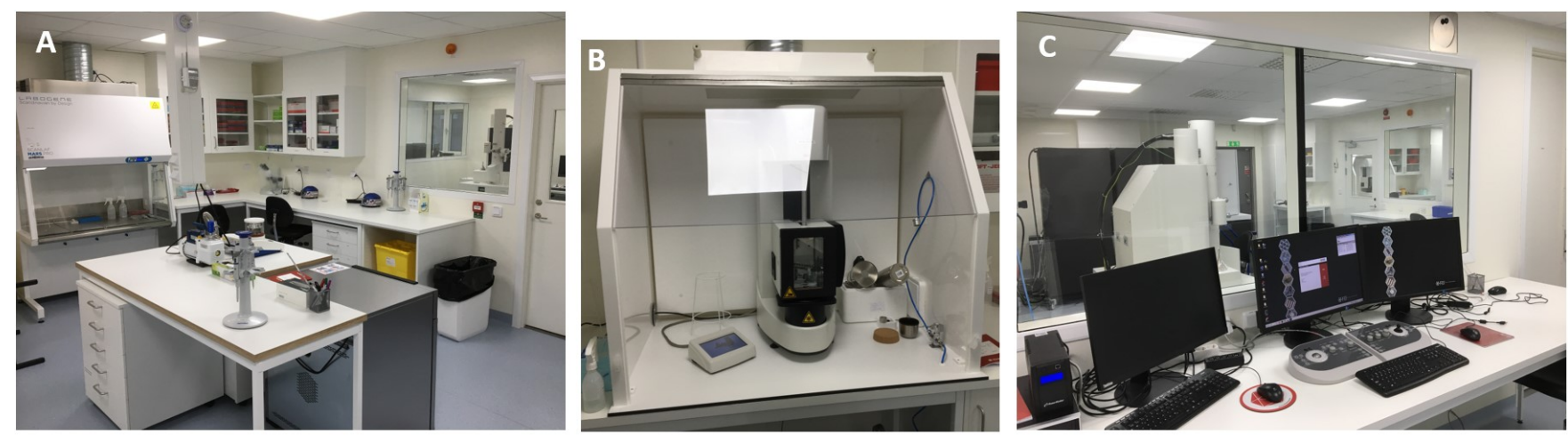

Figure 1. BSL2 TEM facility, with the preparation room including the biosafety cabinet (A), the plunge-freezing station (B), and the TEM control room (C). 